Cathrine Fabricius-Hansen (Oslo)

\title{
Das IDS und die Tiefe der Grammatik. Ein Blick von außen
}

\begin{abstract}
Thema dieses Beitrags sind die komplexen Nominalphrasen im Deutschen, die von außen gesehen unter Umständen monströs anmuten. Ein besonderes, wohl bekanntes Problem bieten dabei sogenannte erweiterte vorangestellte Attribute. Die Komplexitäten geben u.A. zu folgenden Fragen Anlass: Inwiefern lässt sich die ,Ausuferung‘ der deutschen Nominalphrase funktional begründen? Falls es ein Rationale hinter den Komplexitäten gibt, wie lösen dann Sprachen, die entsprechende Ausbaumöglichkeiten nicht besitzen, die einschlägigen funktionalen Aufgaben? Hier soll primär die erste Frage diskutiert werden anhand von authentischen Text(ausschnitt)en, die das Zusammenspiel zwischen vorangestellten und nachgestellten ,Erweiterungen' der Nominalphrase - Relativsätze eingeschlossen - wie auch die Funktion sogenannter nichtrestriktiver Attribute im Diskurs veranschaulichen können; die zweite Frage wird in relevanten Zusammenhängen mit berücksichtigt.
\end{abstract}

\section{Vorbemerkung}

Die Gelegenheit, zum 50. Jahrbuch des IDS beitragen zu dürfen, möchte ich einleitend dazu nutzen, darauf hinzuweisen, was das IDS über die Jahre alles für die germanistische Lingustik im Ausland getan hat:

- Die Erstellung umfassender empirischer Beschreibungen der deutschen Sprache, auf die wir, die Auslandsgermanisten, haben zugreifen können (die „Grammatik der deutschen Sprache“, das „Handbuch der deutschen Konnektoren“, unzählige Einzeluntersuchungen - das ganze elektronische Grammatiksystem grammis, Lexika und Wörterbücher, ...).

- Eine Reihe kontrastiver Grammatiken und jetzt auch eine breitere typologisch basierte Beschreibung des Deutschen im europäischen Vergleich.

- Die Erstellung und Bereitstellung elektronischer Korpora;

- Eine hervorragende Bibliothek und dank der großen Gastfreundlichkeit des Instituts auch hervorragende Möglichkeiten für ausländische Kolleg/innen, sie zu nutzen.

- Fünfzig Jahrestagungen (von denen ich selber wahrscheinlich ungefähr 35 beigewohnt habe) und unzählige kleinere Workshops und Kolloquien zu wechselnden Themen. 
Dem Direktor als Vertreter des Instituts - und stellvertretend auch für frühere Direktoren - sei an dieser Stelle im Namen der linguistischen Germanist/innen im Ausland herzlich dafür gedankt.

\section{Allgemeines}

Der Titel dieses Beitrags ist eine Anspielung auf den 1951 erschienenen Roman „Die Strudlhofstiege“ von Heimito von Doderer, dessen vollständiger Titel wie folgt lautet: „Die Strudlhofstiege oder Melzer und die Tiefe der Jahre“. Der Roman spielt in Wien, wo sich auch die Strudlhofstiege befindet, die an zentralen Stellen in der Handlung mitspielt. Sie ist unten abgebildet (Abb. 1).

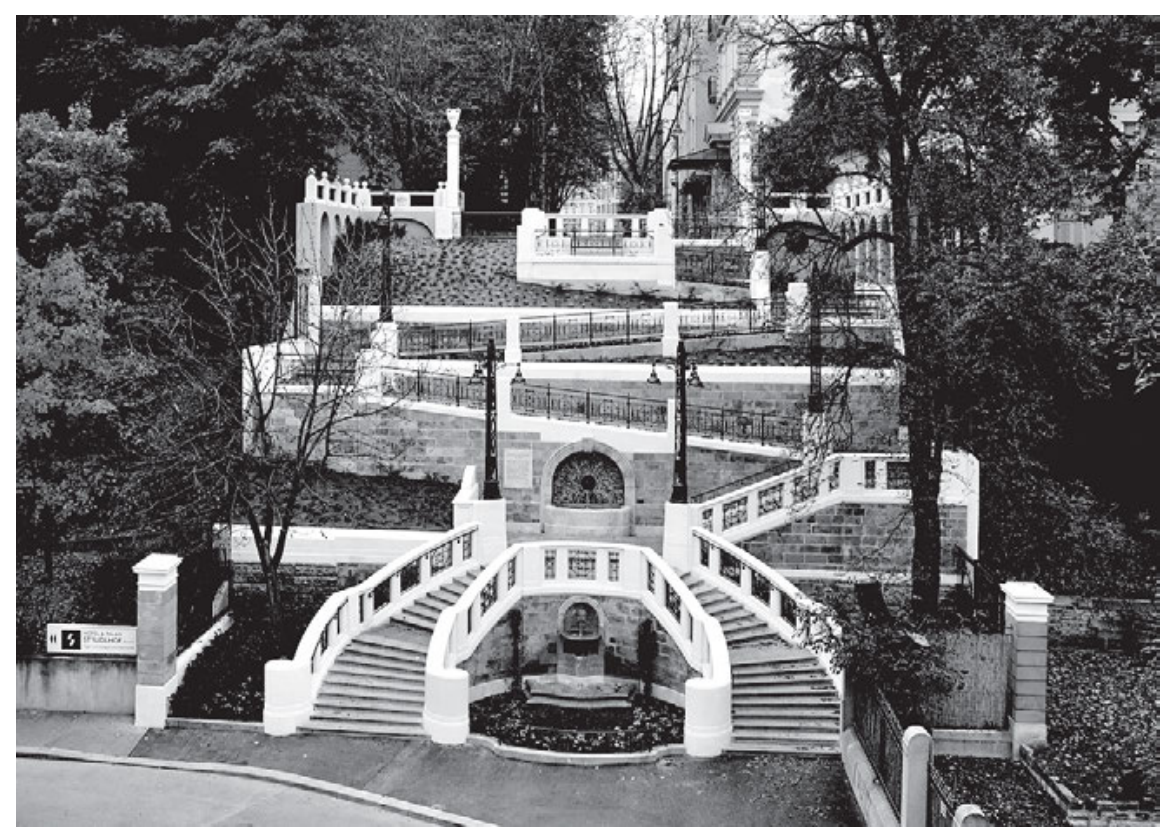

Abb. 1: Die Strudlhofstiege in Wien

Die Leserin/der Leser möge sich einen Moment überlegen, wie sie/er diese Treppe, diese Einrichtung, beschreiben würde - wie der ganze Prozess verläuft, von dem Augenblick an, wo sie/er das Foto (stellvertretend für die Treppe selber) sieht und die Aufgabe bekommt, sie zu beschreiben, bis das sprachliche Produkt, sagen wir in schriftlicher Form, vorliegt. 
Wolfgang Klein zerlegt im Aufsatz „Zwei Leitgedanken zu ,Sprache und Erkenntnis““ diesen Prozess in folgende Schritte (Klein 2007; siehe auch Stutterheim/Klein 2002):

Zuerst bildet sich eine im Gedächtnis gespeicherte „Gesamtvorstellung“, auch „Sachkomplex“ genannt, durch kognitive Vorgänge bestimmt, die intensiv erforscht werden, auf die ich hier jedoch nicht eingehen kann. Es handelt sich nach Klein (2007, S. 31) um „eine Menge von Informationen, die im Kopf des Sprechers gespeichert sind und nun zum Zweck der sprachlichen Umsetzung abgerufen werden“.

Aus diesem Sachkomplex muss dann eine Auswahl (,Selektion“) getroffen werden - man kann und sollte eben nicht alles sagen. Die Selektion wird zum Teil zweck-, situations- und adressatenbestimmt sein, im konkreten Fall unter anderem dadurch, wie man die Aufgabe auffasst oder sich zurechtlegt - etwa als Wegbeschreibung (von oben oder unten?) oder als Beschreibung aus der Vogelperspektive -, ob die Adressaten die Treppe auch sehen, usw. Das heißt, es muss ein übergeordneter „Plot“ der Darstellung - eine „Quaestio“ - festgelegt und dabei auch entschieden werden, wie detailliert vorzugehen ist: die „Granularität“ der Beschreibung muss bestimmt werden. ${ }^{1}$

$\mathrm{Zu}$ entscheiden ist auch, inwieweit die ausgewählte Information durch Informationen anderer Art („Additionen“) - „Vergleiche $\mathrm{zu}$ anderen Vorgängen, Querverweise, emotionale Stellungnahme, Bekundungen der Unsicherheit, Kommentare, Bewertungen, moralische Urteile und dergleichen mehr“ (Klein 2007, S. 32) angereichert werden soll. Entschieden werden muss ferner, in welcher Reihenfolge die einzelnen Bestandteile der ausgewählten Information zur Sprache gebracht werden wollen. Das heißt, wie die „Linearisierung“ zu erfolgen hat.

Das Ergebnis dieser verschiedenen Schritte/Entscheidungen nennt Klein die „Diskursrepräsentation“: „all jene Informationen, die der Sprecher in einer bestimmten Anordnung tatsächlich in Worte kleiden will“" (Klein 2007, S. 31). ${ }^{2}$ Diese liegt dann der sprachlichen „Formulierung“ zu Grunde.

Der Prozess ist sehr summarisch in Abbildung 2 zusammengefasst.

1 Stutterheim/Klein (2002) erwähnen auch „Funktionszuordnung“ („function assignment“) zu Partizipanten als zusätzliche Restriktion auf die Diskursrepräsentation. Sie berührt vor allem die Satzebene und soll hier nicht berücksichtigt werden.

2 Der Kleinsche Begriff der Diskursrepräsentation ist verwandt, aber nicht identisch mit dem Begriff, wie er in der sogenannten Diskursrepräsentationstheorie (siehe Kamp/Reyle 2011) verstanden wird. 


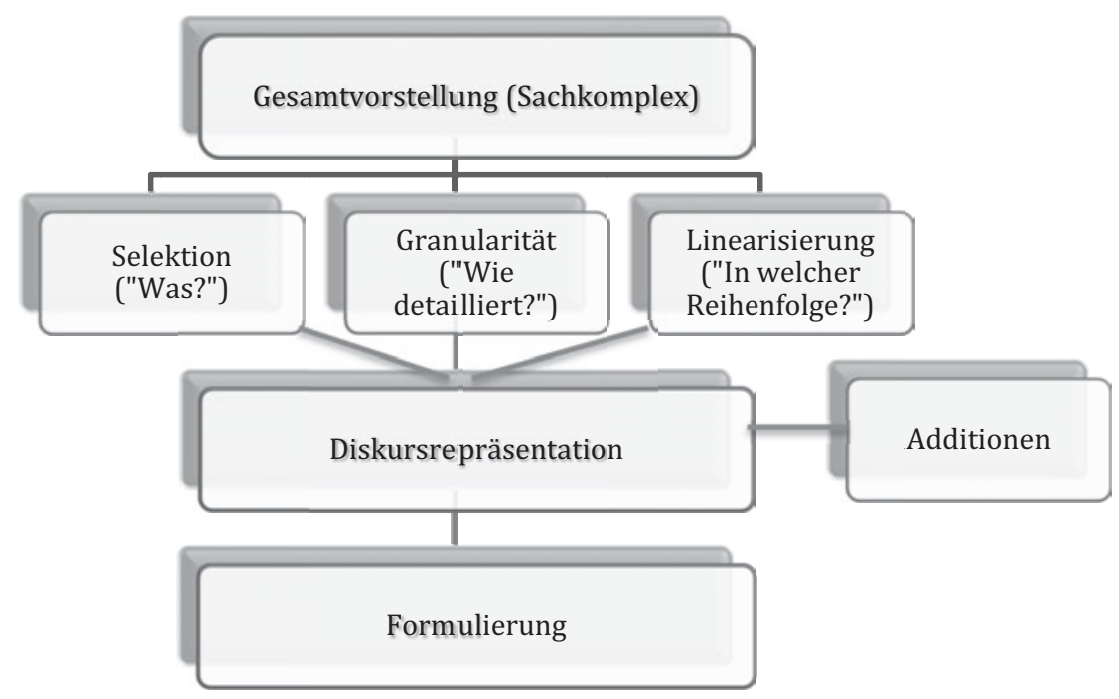

Abb. 2: Vom „Sachkomplex“ zur „Diskursrepräsentation“

Beim spontanen Reden werden viele Entscheidungen online getroffen, und zwar bei jedem Schritt in Abhängigkeit von dem, was bis dahin gesagt worden ist; der Sprecher/die Sprecherin hat vielleicht am Anfang sogar keinen klaren Plot. Beim Schreiben hat man hingegen die Möglichkeit, alles zu überarbeiten, um sicherzustellen, dass das sprachliche Produkt, der Text, angemessen ist in dem Sinne, dass sich bei den Adressaten eine Diskursrepräsentation bildet, die derjenigen möglichst ähnlich ist, die dem Text zugrunde liegt. (Man kann leider nie ganz sicher sein, dass das gelingt!) (Siehe dazu Zeevat 2014).

Eine brisante Frage ist nun, an welchem Punkt im Prozess von der Gesamtvorstellung zur Formulierung sprachspezifische Einflüsse sich geltend machen - d.h. inwiefern nicht nur die Endformulierung (selbstverständlich), sondern auch die Selektion, die Granularität und die Linearisierung (auf Diskurs-/Textebene) sowie die Additionen durch die jeweilige Sprache mitbedingt sind. Klein und Stutterheim (unter Anderen) sprechen dabei von „sprachlicher Perspektivierung“ (Klein 2007; Stutterheim/Klein 2002).

Dass der Wortschatz in diesem Zusammenhang eine Rolle spielt, liegt auf der Hand - gehen doch Sprache und Begriffsbildung gewissermaßen Hand in Hand. Aber wie steht es um grammatische Eigenschaften der Sprachen? Inwiefern ist die Grammatik in nicht trivialer Weise dafür mit entscheidend, was aus dem Sachkomplex für die Versprachlichung ausgewählt wird und wie das zu Versprachlichende auf Diskursebene linearisiert wird? 
Untersuchungen zum Zweit-/Fremdspracherwerb, die über längere Zeit in der Heidelberger Gruppe um Christiane von Stutterheim durchgeführt worden sind, deuten darauf hin, dass eine solche grammatikbedingte sprachliche Perspektivierung tatsächlich stattfindet, zumindest in bestimmten Bereichen (Tempus, Aspekt, ...); siehe z.B. Caroll et al. (2008); Stutterheim et al. (2012); Stutterheim/ Carroll (2007); Stutterheim/Klein (2002).

Interessant aus sprachvergleichender Sicht ist nicht zuletzt die Frage, welchen Einfluss zwei grammatische Charakteristika des Deutschen auf die Textgestaltung ausüben:

1. Die grundlegende Rechtsköpfigkeit (OV-Linearisierung, Verbendstellung) des Deutschen auf Satz-/Verbalphrasenebene.

2. Die (damit zusammenhängende) Möglichkeit, die Nominalphrase nicht nur mit Hilfe von Präpositionalattributen, Relativsätzen und Appositionen nach rechts (postnominal) zu erweitern, wie es auch in den romanischen Sprachen und in den linksköpfigen germanischen Sprachen möglich ist, sondern auch mit Hilfe sogenannter erweiterter vorangestellter Adjektiv- und Partizipialattribute sowie Attributreihungen nach links (pränominal) kräftig auszubauen; vgl. Fabricius-Hansen (2010a, 2010b); Schmidt (1993); Struckmeier (2007); Weber (1994).

Die folgende Diskussion wird sich auf Fragen konzentrieren, die mit dem zweiten Problemkreis zusammenhängen:

- Welche Funktionen haben sogenannte nichtrestriktive Attribute - in erster Linie vorangestellte Attribute und Relativsätze - im Textzusammenhang? Inwiefern unterscheiden sich (erweiterte) vorangestellte Attribute und Relativsätze in dieser Hinsicht voneinander

- Welchen Einfluss hat die pränominale Ausbaufähigkeit der Nominalphrasen im Deutschen auf die Textgestaltung (im Vergleich etwa zum Englischen oder Norwegischen)? Oder allgemeiner: Wie wirken sich sprachspezifische Beschränkungen des prä- und/oder postnominalen Ausbaus von Nominalphrasen auf die Textgestaltung aus?

Ich werde diese Fragen allerdings nicht systematisch und schon gar nicht erschöpfend behandeln (dafür reichen weder der Platz noch meine Einsicht aus), sondern nur noch einige zentrale Punkte anhand ausgewählter Textbeispiele veranschaulichen. Da es dabei vor allem um sogenannte nichtrestriktive Attribute geht, soll dieser Begriff zunächst kurz erläutert werden (Abschnitt 3). Abschnitt 4 und Abschnitt 5 befassen sich aus etwas unterschiedlicher Perspektive mit diskursfunktionalen Aspekten nichtrestriktiver Attribute in definiten Nominalphrasen. In Abschnitt 6 wird Bilanz gezogen. 


\section{Restriktive und nichtrestriktive Attribute in definiten Nominalphrasen}

Betrachten wir den Textauszug in (1) - den narrativen Anfang des Romans „Der Turm“.

\section{(1) AUFFAHRT}

(a) Die elektrischen Zitronen aus dem VEB „Narva“, mit denen der Baum dekoriert war, hatten einen Defekt, flackerten hin und wieder auf und löschten die elbabwärts liegende Silhouette Dresdens. (b) Christian zog die feucht gewordenen, an den wollenen Innenseiten mit Eiskügelchen bedeckten Fäustlinge aus und rieb die vor Kälte fast taub gewordenen Finger rasch gegeneinander, hauchte sie an - (c) der Atem verging als Nebelstreif vor dem finster liegenden, in den Fels gehauenen Buchensteig, der hinauf zu Arbogasts Instituten führte. Die Häuser der Schillerstraße verloren sich im Dunkel; vom nächstliegenden, einem Fachwerkhaus mit verriegelten Fensterläden, lief eine Stromleitung ins Geäst einer der Buchen über dem Felsdurchgang, ein Adventsstern brannte dort, hell und reglos. (d) Christian, der über das Blaue Wunder und den Körnerplatz gekommen war, ging weiter stadtauswärts, in Richtung Grundstraße, und erreichte bald die Standseilbahn. (Uwe Tellkamp: Der Turm. Frankfurt a.M. 2008, S. 15; Hervorhebungen CF)

An diesem Textauszug lässt sich illustrieren, was ich unter einem „nichtrestriktiven Attribut“ verstehen möchte: Ein Attribut, das zur Identifikation des von der Nominalphrase eingeführten/bezeichneten Diskursreferenten (Kamp/Reyle 2011) nichts beiträgt und in dem Sinne (syntaktisch und) semantisch entbehrlich (,eliminable“, Schlenker 2013) ist. Das heißt, nichtrestriktive Attribute enthalten zusätzliche Information über einen schon identifizierten bzw. ohne das Attribut identifizierbaren Referenten. (Siehe zum Restriktivitätsbegriff, der vorwiegend im Zusammenhang mit Relativsätzen diskutiert worden ist, unter Anderen Blühdorn (2007); Fabricius-Hansen (2009); Holler (2008; 2011); Lötscher (1998); Umbach (2006); Zifonun (2001); Zifonun/Hoffmann/Strecker (1997).

In unserem Textausschnitt finden sich drei Relativsätze (RSe, kursiv) und sieben erweiterte vorangestellte Attribute (EVAs, kursiv und unterstrichen).

Die ersten beiden Relativsätze - jeweils in (a) und (c) - sind restriktiv: Man braucht sie, um zu verstehen, von welchen elektrischen Zitronen bzw. welchem Buchensteig die Rede ist; es handelt sich um „propositional“ bzw. „lokal verankernde Modifikationen“ im Sinne von (Zifonun 2010). Buchensteig in (d) könnte 
allerdings auch ein Name sein, in welchem Fall der Relativsatz nichtrestriktiv ist. Mit anderen Worten: Ob der RS als restriktiv oder nichtrestriktiv aufgefasst wird, hängt vom Wissen der Leser/innen ab. Ähnliches gilt für das vorgestellte Partizipialattribut in der Nominalphrase die elbabwärts liegende Silhouette Dresdens in (a). Um zu entscheiden, ob es von dort aus gesehen, wo der im nächsten Satz eingeführte Protagonist Christian sich gerade befindet, nur eine elbabwärts oder auch eine elbaufwärts liegende Silhouette Dresdens geben kann (der norwegische Übersetzer des Romans hat die Textstelle anscheinend im zweiten Sinne verstanden, im Unterschied zum englischen) - um das entscheiden zu können, muss man sich als Leser in Dresden auskennen. Allerdings handelt es sich hier um den Anfang der Erzählung, und da macht umgekehrt die Auskunft, dass die Silhouette Dresdens aus der Sicht des Protagonisten elbabwärts liegt, dem kundigen Leser klar(er), wo der Protagonist sich in etwa befinden könnte (siehe Abschnitt 4). - Die EVAs in (b) und (c) sowie der Relativsatz in (d) sind alle zweifelsfrei nichtrestriktiv.

Fassen wir den Relativsatz in (d) als restriktiv und elbabwärts liegende in (a) als nichtrestriktiv auf, so erhalten wir, wenn wir die nichtrestriktiven Attribute auslassen, einen Text (1'), dessen definite Nominalphrasen referenziell wie im Originaltext (1) interpretiert werden und der auch sonst kohärent erscheint (siehe jedoch Kommentar zu (d) in Abschnitt 4).

(1') [a] Die elektrischen Zitronen aus dem VEB „Narva“, mit denen der Baum dekoriert war, hatten einen Defekt, flackerten hin wieder auf und löschten die Silhouette Dresdens. [b] Christian zog die Fäustlinge aus und rieb die Finger rasch gegeneinander, hauchte sie an - [c] der Atem verging als Nebelstreif vor dem Buchensteig, der hinauf zu Arbogasts Instituten führte. Die [...] reglos. [d] Christian ging weiter stadtauswärts, in Richtung Grundstraße, und erreichte bald die Standseilbahn.

Angesichts der syntaktischen und semantischen Entbehrlichkeit der nichtrestriktiven Attribute stellt sich die Frage, wozu sie dann überhaupt da sind - welche Funktionen die in ihnen enthaltenen Informationen haben (Abschnitt 4) - und warum diese Informationen nicht etwa in Form selbstständiger Sätze gebracht werden (Abschnitt 5). 


\section{Eine diskursfunktionale Perspektive auf nichtrestriktive Attribute in definiten Nominalphrasen}

\subsection{Nichtrestriktive Attribute im Beispiel (1)}

In Abschnitt 2 wurde darauf hingewiesen, dass kohärenten Text(ausschnitt)en nach Klein, Stutterheim und anderen eine Quaestio zugrunde liegt, d.h. eine übergeordnete Frage, die der Text beantworten soll und die demzufolge zumindest teilweise die Textentfaltung steuert. Für diesen oder eng verwandte Begriffe findet man auch den Terminus Question under Discussion (QuD), den ich im Folgenden als Synomym zu Quastio verwenden werde (siehe zu QuD etwa Onea 2013).

Das erste Kapitel des Romans, dem unser Textausschnitt entnommen ist, folgt dem Protagonisten Christian auf seinem Weg nach Hause durch das nächtliche Dresden. Als übergeordnete Quaestio wäre demnach etwa „Was tut/tat Christian? Wie verläuft/verlief seine Wanderung/Fahrt?“ anzusetzen. Diese QuD wird durch die chronologisch fortschreitende Erzählung beantwortet. Eingeschoben zwischen den einzelnen Schritten des Hauptstrangs - der Narration - finden sich Beschreibungen dessen, was Christian auf seiner Wanderung sieht, als Hintergrund der Narration (oder als zweite Hauptstruktur).

In dem um die nichtrestriktiven Attribute gekürzten Text (1') leitet der Satz (1'b) Christian zog die Fäustlinge aus und rieb die Finger rasch gegeneinander, hauchte sie an die Beantwortung der oben genannten narrativen Quaestio - die „main story line“ (Klein/Stutterheim 1991) - ein. Genauso verhält es sich natürlich im Originaltext (1b). Die Narration wird vom nächsten mit Christian eingeleiteten Satz (1d) - Christian [...] ging weiter stadtauswärts, in Richtung Grundstraße, und erreichte bald die Stadtseilbahn. - weitergeführt.

Die Satz- oder Verbalphrasenreihe in (1b) beschreibt eine Handlungskette (die Fäustlinge ausziehen, die Finger reiben, sie anhauchen), die als eine komplexe Handlung mit dem Zweck, die Finger zu wärmen, verstanden werden kann. Die nichtrestriktiven Attribute tragen dazu - und das heißt zur Beantwortung der Quaestio - nicht direkt bei. Sie beantworten aber eine Frage, die sich im gegebenen Zusammenhang leicht erhebt, - eine untergeordnete Frage, die sozusagen vorweggenommen wird (vgl. Onea 2013): Warum tut Christian das alles? Warum sind Christians Finger so kalt geworden, dass diese komplexe Handlung nötig ist? Das heißt, die nichtrestriktiven Attribute beschreiben eine handlungsauslösende Kausalkette - den kausalen Hintergrund von Christians Handlung(en). 
Zusammen machen die nichtrestriktiven Attribute in (1b) deutlich, dass es in der aktuellen Nacht sehr kalt ist, ohne dass das irgendwo explizit gesagt worden wäre, und tragen so zur Beschreibung des Hintergrunds im weiteren Sinne bei.

Eine ähnliche Funktion könnte man den vorangestellten Attributen in dem Satz (1c) (- der Atem verging als Nebelstreif vor dem finster liegenden, in den Fels gehauenen Buchensteig, der hinauf zu Arbogasts Instituten führte) zuschreiben: Sie erklären, warum Christians Atem so deutlich als Nebelstreif zu sehen ist, können aber auch einfach als gegenstandsbezogene Elaborierung gedeutet werden.

Im Satz (1d), der die narrative Hauptstruktur fortführt (Christian, der über das Blaue Wunder und den Körnerplatz gekommen war, ging weiter stadtauswärts, in Richtung Grundstraße, und erreichte bald die Standseilbahn.) kommt ein nichtrestriktiver Relativsatz vor, der wie die Attribute in (1b) eine durch den Satz nahegelegte Frage (,Woher war Christian gekommen?“) beantwortet. So wird auch - für Leser/innen mit passendem Wissen - verständlich(er), was mit der im Trägersatz vorkommenden Richtungsangabe weiter konkret gemeint ist.

Im Anfangssatz (1a) Die elektrischen Zitronen aus dem VEB „Narva [...] flackerten hin und wieder auf und löschten die elbabwärts liegende Silhouette Dresdens dient das vorangestellte Attribut elbabwärts liegende, nichtrestriktiv gedeutet, gleichfalls indirekt zur Bestimmung vom Standort des Protagonisten, durch dessen Augen alles gesehen wird, - allerdings bevor dieser überhaupt eingeführt worden ist.

\subsection{Diskursfunktion und ,Entbehrlichkeit`}

In Abschnitt 3 wurden nichtrestriktive Attribute als entbehrlich charakterisiert in dem Sinne, dass sie für die Identifikation des von der aktuellen Nominalphrase ,einführten‘ Diskursreferenten nicht nötig seien. Der Vergleich von (1) und der ,abgespeckten' Variante (1') hat gezeigt, dass nichtrestriktive Attribute auch in dem etwas weiteren Sinne entbehrlich sein können, dass der Text nach ihrer Auslassung kohärent bleibt. Das ist jedoch nicht immer so: Unter Umständen enthalten nichtrestriktive Attribute eine Information, die für die Interpretation von sprachlichen Elementen im (nachfolgenden Teil des) jeweiligen Matrixsatz(es) direkt relevant oder sogar notwendig ist, so dass ihre Auslassung den Text inkohärent oder weniger verständlich macht (vgl. Lötscher 1998; Peyer 1997 für Relativsätze). Man vergleiche etwa (1'd) mit (1d), wo der Relativsatz zum Verständnis von weiter dient (siehe Abschnitt 4.1), und auch (2') mit (2), wo der RS zur Erfüllung der Voraussetzung beiträgt, die man typisch mit dem Begriff ,finden` verbindet, nämlich dass man etwas sucht. 
(2) [...] Wiesenthal, der einen Mann namens Schulz in Frankfurt sucht, macht das Telefonbuch auf, findet einen Schulz, dessen Gattin Heidrun heiß, und ist überzeugt: „Das ist er.“ Und meistens ist er es. (Simon Wiesenthal: Rede, nicht Rache. Frankfurt a.M. 1988, S. 60)

(2') [...] Wiesenthal macht das Telefonbuch auf, findet einen Schulz, dessen Gattin Heidrun heiß, und ist überzeugt: „Das ist er.“ [...]

In den genannten Fällen sind die Atttribute, ob entbehrlich oder nicht, lokal relevant, d.h. deutlich auf die (Sub-)Quaestio, die der aktuelle Satz (mit) beantwortet, bezogen, indem sie Fragen vorwegnehmen, die sich natürlich daraus ergeben (vgl. Onea 2013 für Appositionen); vgl. (1b-d).

In anderen Fällen ist das Attribut lokal nicht relevant und insofern durchaus entbehrlich, trägt jedoch in indirekter Weise zur Beantwortung der übergeordneten Quaestio bei. Das EVA elbabwärt liegende in (1a) war ein Beispiel dafür. Ein weiteres Beispiel bietet (3).

(3) Dass Elstern intelligent sind und neben einer herausragenden Beobachtungsgabe auch über technisches Geschick verfügen, wir auch von ihren menschlichen Feinden anerkannt. Sie sind zum Beispiel in der Lage, Dachziegel anzuheben, um darunter portionierte Nahrung zu verstecken und bei Bedarf wieder hervorzuholen. Zum Vorteil wurde das dem schwarz-weißen Vogel mit dem metallic-schwarzblau glänzenden langen Schwanz aber nie angerechnet. Im Gegenteil. Die Geschichte ihrer Bestandsentwicklung im 20. Jahrhundert in Deutschland spiegelt auf merkwürdige Weise die politische Kultur des Landes. (Cord Riechelmann: Krähen. Berlin, S. 48; Hervorhebung: CF)

Die Informationen über die Farbe der Elster und das Aussehen ihres Schwanzes ist im aktuellen Zusammenhang irrelevant - die Frage „Wie sieht die Elster und insbesondere ihr Schwanz denn aus?" fällt einem beim Lesen des ,abgespeckten“ Satzes Zum Vorteil wurde das dem Vogel aber nie angerechnet nicht unmittelbar ein. Diese Informationen gehören aber natürlich zu einer Gesamtbeschreibung von Elstern mit dazu, auch wenn ihr Verhalten eher als ihr Äußeres im Fokus steht, wie es im aktuellen Text der Fall ist.

In wieder anderen Fällen handelt es sich bei nichtrestriktiven Attributen um Einstellungsbekundungen, Bewertungen, metatextuelle Hinweise oder Kommentare anderer Art von Seiten des Autors bzw. der Autorin, d.h. nach Klein (2007) um „Additionen“, die eindeutig nicht zur Hauptstruktur des Textes gehören und insofern entbehrlich sind, die aber aus anderen Gründen für das Textverständnis 
wichtig sein können (siehe beispielsweise Lötscher 1998; Loock 2010 für nichtrestriktive Relativsätze).

\subsection{Zusammenfassung}

Unsere Betrachtungen lassen sich wie folgt zusammenfassen:

- Ein nichtrestriktives Attribut kann, obwohl es ,eigentlich‘ zur Nominalphrase gehört, lokale ereignis- oder sachverhaltsbezogene Relevanz haben, indem es entweder direkt für die Interpretation der Verbalphrase verwertet wird, wie in (1d) und (2), oder indirekt den (eventuell kausalen) Hintergrund des im Satz beschriebenen Geschehens oder Sachverhalts beisteuert und so die Granularität der Beschreibung erhöht, wie in (1b-c).

- Das Attribut kann Information enthalten, die keine lokale ereignis- oder sachverhaltsbezogene Relevanz besitzt, aber eventuell, wie in (1a) und (3), indirekt zur Beantwortung der übergeordneten Quaestio beiträgt.

- Das Attribut kann schließlich eindeutig eine „Addition“ sein, die weder in der einen noch in der anderen Hinsicht für die Hauptstruktur relevant ist (siehe z.B. Lötscher 1998).

- Nichtrestriktive Attribute sind zwar definitionsgemäß (Abschnitt 3) für die Referentenidentifikation entbehrlich, können aber für die Textkohärenz unverzichtbar sein oder allgemein das Textverstehen erleichtern.

\section{Nichtrestriktives Attribut oder selbstständiger Satz?}

Man kann sich fragen, warum die in nichtrestriktiven Attributen enthaltene Information in dieser Form und nicht etwa in Form von selbstständigen Sätzen realisiert (siehe Abschnitt 3) wird. Zu dieser Frage liefert Andreas Lötscher im Aufsatz „Die textlinguistische Interpretation von Relativsätzen“ (1998) mit Bezug auf Relativsätze wichtige Einsichten:

- Die Linearisierung und damit auch die Verarbeitung verlaufen bei nicht-finaler RS-Anschluss anders als bei selbstständigen Sätzen, die im Normalfall nacheinander folgen.

- Eingeschobene parenthetische Sätze haben als syntaktisch und prosodisch ,isolierte' Einheiten einen deutlich anderen Stellenwert als eingebettete Relativsätze. 
- Relativsätze (sogenannte weiterführende Relativsätze ausgenommen) werden aufgrund ihrer attributiven Funktion „thematisch speziell auf den Referenten der dominierenden Nominalgruppe bezogen“ (Lötscher 1998, S. 118), was auf selbstständige Sätze nicht zutrifft.

Ein Relativsatz kann selbst wieder einem untergeordnetem Satz zugeordnet sein, während ein entsprechender Hauptsatz funktional eher auf globaler Textebene diskursfunktional angeschlossen wird (vgl. hierzu auch Schlenker 2013).

Diese Charakterisierung hat mutatis mutandis auch für den Vergleich von nichtrestriktiven vorangestellten Attributen und selbstständigen Sätzen Gültigkeit. Hinzu kommt noch, dass pränominale Attribute anders als Relativsätze dem nominalen Kopf vorangehen und sowohl syntaktisch als prosodisch auch stärker in den Matrixsatz integriert sind als Relativsätze (Fabricius-Hansen i.Dr.). So kann es nicht wundernehmen, dass die ,Heraufstufung der in (1b) vorkommenden EVAs den Text kräftig ändert; vgl. (1b) und (4)-(8).

(1b) Christian zog die feucht gewordenen, an den wollenen Innenseiten mit Eiskügelchen bedeckten Fäustlinge aus und rieb die vor Kälte fast taub gewordenen Finger rasch gegeneinander, hauchte sie an - ...

(4) Christian zog die Fäustlinge aus und rieb die Finger rasch gegeneinander. Seine Fäustlinge waren feucht (geworden) und an den wollenen Innenseiten mit Eiskügelchen bedeckt (worden), seine Finger waren fast taub vor Kälte. Er hauchte sie an - ...

(5) Christian zog die Fäustlinge aus, sie waren feucht (geworden) und an den wollenen Innenseiten mit Eiskügelchen bedeckt. Seine Finger waren fast taub vor Kälte. Er rieb die Finger/sie rasch gegeneinander, hauchte sie an - ...

(6) Christian zog die Fäustlinge aus, sie waren feucht (geworden) und an den wollenen Innenseiten mit Eiskügelchen bedeckt. Er rieb die Finger rasch gegeneinander. Sie waren fast taub vor Kälte. Er hauchte sie an - ...

(7) Christians Fäustlinge waren feucht geworden und an den wollenen Innenseiten mit Eiskügelchen bedeckt. Er zog die Fäustlinge/sie aus und rieb die Finger rasch gegeneinander. Sie waren fast taub vor Kälte. Er hauchte sie an - ...

(8) Christians Fäustlinge waren feucht (geworden) und an den wollenen Innenseiten mit Eiskügelchen bedeckt. Seine Finger waren fast taub vor Kälte. Er zog die Fäustlinge aus und rieb die Finger rasch gegeneinander, hauchte sie an - ... 
In der Originalfassung (1b) haben wir es mit einer Satzreihe $\mathrm{zu}$ tun, die, wie im Abschnitt 4.1 festgestellt wurde, als Ganzes zur erzählenden Hauptstruktur des Textes gehört - ja, diese sogar einleitet. Der kausale Hintergrund wird gewissermaßen auf einer zweiten, untergeordneten Ebene mitgegeben. In (4)-(6) wird die narrative ,main story line، in unterschiedlicher Weise durch Hintergrundbeschreibungen unterbrochen, die teils als Erklärungen nachgeliefert werden, teils die im nachfolgenden Satz beschriebene Handlung vorbereiten. In (7)-(8) wiederum wird der narrative Anfang um einen bzw. zwei Sätze hinausgeschoben, mit der Folge, dass der Protagonist nicht gleich als Agens, als handelnde Person, sondern erstmal als Besitzer oder Träger von Fäustlingen eingeführt wird.

Wir können aus dieser Diskussion folgenden Schluss ziehen: Die Herunterstufung ,hauptsatzfähiger، Information zu komplexen Attributen muss nicht unbedingt der Informationsverdichtung dienen, sie ermöglicht es vielmehr auch, Informationsteile mit unterschiedlicher Diskursfunktion ohne Verlust der Granularität so zu bündeln, daß die ,main story line‘ transparent bleibt. Man vergleiche dazu auch (9), wo das EVA zuvor überall auf dem Land vorkommenden als Hauptsatz formuliert den Übergang vom ersten zum zweiten Absatz stören würde.

[Fortsetzung von (3)]

Die Geschichte ihrer Bestandsentwicklung im 20. Jahrhundert in Deutschland spiegelt auf merkwürdige Weise die politische Kultur des Landes.

Von 1900 bis 1920 waren die zuvor überall auf dem Land vorkommenden Vögel durch massive Verfolgung und Bejagung soweit dezimiert, dass die meisten Ornithologen mit ihrem baldigen Aussterben rechneten. Gegen Ende der 20er Jahre erholten sich die Elsternpopulationen und kehrten in früher verlassene Gebiete zurück. Nach dem zweiten Weltkrieg erreichten sie in den waffenlosen Jahren bis 1950 wieder Siedlungszahlen, wie man sie aus der Mitte des 19. Jahrhunderts kannte. Um nach 1950 mit der wieder einsetzenden Jagd und der zunehmenden Intensivierung der Landwirtschaft abermals unter massiven Druck zu geraten.

Hinzu kommt, dass vorangestellte Attribute in definiten Nominalphrasen aus Gründen, auf die ich hier nicht eingehen kann, sehr wohl ,Information“ enthalten können, die diskurs- und präsumptiv dann auch adressaten-alt oder zumindest aus dem Kontext leicht erschließbar ist, während man von selbstständigen Sätzen erwartet, dass sie Neues - d.h. Information im echten Sinne - zu bieten haben. In dieser Hinsicht scheinen sich auch vorangestellte Attribute und Relativsätze zu unterscheiden; siehe (Schmidt 1993). Man vergleiche das vorangestellte Attribut in (10) mit dem entsprechenden Relativsatz in (10') und dem parenthetischen 
Hauptsatz in (10”). In der Originalfassung (10) kann man - und wird man wohl - die Charakterisierung der Rufe als „heiser laut“ als aus dem Vorkontext erschließbare und insofern bekannte Information auffassen, in (10') und (10’) wird sie hingegen eher als neu präsentiert.

(10) Es schienen wirklich Himmelsschreie zu sein. Es waren hastig, wie atemlos aufeinanderfolgende kurze hohe ,arr arr"-Rufe, die in einem mehrstimmigen Chor über den Bäumen an der Kindertagesstätte am VictoriaPark in der Methfesselstraße im Berliner Stadtteil Kreuzberg erschallten. Da der Himmel wolkig verhangen war und man deshalb keine Vögel sehen konnte, hätte es sein können, dass die hektisch lauten Rufe von oben in den Wolken ziehenden Vögeln kamen. (C. Riechelmann: Krähen. Berlin 2013, S. 15)

(10’) [...] hätte es sein können, dass die Rufe, die hektisch laut waren, von oben in den Wolken ziehenden Vögeln kamen.

(10’) [...] hätte es sein können, dass die Rufe - sie waren hektisch laut- von oben in den Wolken ziehenden Vögeln kamen.

Das heißt, in vorangestellten Attributen können Sprecher Information unterbringen, die je nach Adressaten alt bzw. erschließbar oder neu sein kann; sie müssen sich nicht um den Status so kodierter Information kümmern. Insofern scheinen pränominale Attribute bei einem anonymen und/oder heterogenen Adressatenkreis besser als Relativsätze möglichen Verstehensdefiziten vorbeugen zu können, ohne Leser/innen, die solche Hilfe nicht nötig haben, zu irritieren (FabriciusHansen 2009, i.Dr.).

Es kann noch hinzugefügt werden, dass der parallele Ausbau der Nominalphrase nach links und nach rechts die Möglichkeit eines diskursfunktionalen Zusammenspiels zwischen den beiden Attributtypen eröffnet. Dies lässt sich am Beispiel (11) veranschaulichen, wo der Relativsatz die im vorangestellten Attribut ausgedrückte Charakterisierung des Vogelhirns näher präzisiert.

(11) So konnte kürzlich die Arbeitsgruppe um den Psychologen Helmut Prior von der Frankfurter Goethe-Universität nachweisen, dass sich Elstern im Spiegel erkennen können. Was bisher ein Privileg von Elefanten, Schimpansen, Delfinen und Menschenkindern war, muss nun auch in der von der menschlichen Abstammungslinie sehr weit entfernten Vogellinie verortet werden.

Dass das völlig anders organisierte Vogelhirn, das keine organisierend integrierende Struktur wie die Großhirnrinde, den Neokortex, aufweist, in der Lage ist, ähnliche kognitive Fähigkeiten vorzubringen, 
kann man auch als eine Absage an stammesgeschichtliche Fortschrittsvorstellungen sehen. (C. Riechelmann: Krähen. Berlin 2013, S. 56-57; Hervorhebungen: $\mathrm{CF}$ )

\section{Bilanz - und der Blick von außen}

Vieles von dem, was in diesem Beitrag über die Diskursfunktion nichtrestriktiver Attribute gesagt wurde, ist mit Bezug auf Relativsätze schon von anderen Forschern in anderen Worten und in einem anderen theoretischen Rahmen festgestellt worden. So unterscheidet Lötscher (1998) „lokale [i.e. auf den Matrixsatzbezogene)] akzeptanzunterstützende“ Relativsätze, die als Begründungen für die Richtigkeit der Matrixsatzbehauptung oder des Mitteilungsakts dienen können, von Relativsätzen, die „Nebeninformationen“ unterschiedlicher Art enthalten; und er erklärt sehr präzise, warum nichtrestriktive Relativsätze sich aus textfunktionaler Sicht oft nicht zu selbstständigen Sätzen oder Parenthesen ,heraufstufen` lassen (vgl. Abschnitt 5 oben). Ähnliche Überlegungen zu nichtrestriktiven (,appositiven“) Relativsätzen im Englischen bietet Loock (2010). Gloning und Seim (Gloning/Seim i.Dr.) präsentieren hochinteressante Beobachtungen zur Diskursfunktion von Attributen in unterschiedlichen Textsorten. Einen spannenden Einblick in das literarische Wirkungspotenzial von Attributkonstruktionen (und anderen Klammerbildungen) bieten widerum Bettens Betrachtungen zu den „Kerkerstrukturen“ in Thomas Bernhards Prosawerken (Betten 2011).

Insgesamt sind die Diskursfunktionen und der Informationsstatus vorangestellter Attribute sowie die Interaktion zwischen vorangestellten und nachgestellten Attributen jedoch meines Wissens nicht in gebührendem Ausmaß untersucht worden, und zwar weder empirisch noch im Hinblick auf die Folgen, die sich daraus für elaborierte und z.T. formalisierte Theorien zur Diskursstruktur wie die Rhetorical Structure Theory von (Mann/Thompson 1988) und die Segmented Discourse Representation Theory (Asher/Lascarides 2003) ergeben könnten.

Unsere Überlegungen haben gezeigt, dass komplexe Attribuierung dazu dienen kann, Informationen, die für die Quaestio - die Hauptstruktur - des Textes oder Textausschnittes keine direkte Relevanz besitzen, so unterzubringen, dass sie die Hauptstruktur nicht verschleiern. ${ }^{3}$ Es kann sich dabei um Informationen handeln, die die Granularität der Darstellung erhöhen oder das Textverständnis

3 Sogenannte weiterführende Relativsätze, die hier unberücksichtigt geblieben sind, bilden in dieser Hinsicht eine Ausnahme (Holler 2008). 
fördern, oder um Informationen, die „Nebenstrukturen“ (Klein 2007) bilden - Einstellungsbekundungen, Bewertungen, Kommentare unterschiedlicher Art. (Dem würde entsprechen, dass beispielsweise die Konturen der Treppe in der Abbildung der Strudlhofstiege (siehe Abschnitt 2) deutlich hervorgehoben würden.) Kann oder will man sich der syntaktischen Herunterstufung nicht bedienen, so gibt es im Prinzip zwei Optionen: Man kann auf Informationen der betreffenden Art verzichten (d.h., sich mit etwa mit den Konturen der Treppe begnügen), um die Transparenz der Hauptstruktur zu sichern. Oder man bringt die zusätzlichen Informationen in Hauptsätzen/selbstständigen Sätzen unter (d.h., beispielsweise den Kontrast zwischen Treppe und Umgebung mindern), mit den negativen Folgen, die das für die Transparenz der globalen Textstruktur haben wird. Der Text wird dann durch einen Wechsel zwischen Sätzen geprägt sein, die sich in unterschiedlicher Weise zur übergeordneten Textfrage verhalten und bei denen man nicht immer weiß, wie sie diskursstrukturell einzuordnen sind (Fabricius-Hansen 2010); vgl. (4)-(8).

Dies sind die aktuellen Optionen für die Sprecher von Sprachen, die - wie Norwegisch - aus system- und/oder normbezogenen Gründen weniger Spielraum für komplexe Attribuierung oder ähnliche Mittel aufweisen. Dass solche durch das grammatische System und/oder grammatische Normen bedingten Beschränkungen etwa beim Übersetzen aus dem Deutschen ins Norwegische Probleme bereiten, wissen wir (Fabricius-Hansen 2010a, 2010b; Solfjeld 2004). Brisanter ist die Frage, inwieweit die Beschränkungen sich schon bei der Selektion aus dem Sachkomplex/der Gesamtvorstellung geltend machen, d.h. die Frage nach den möglichen Konsequenzen von Attribuierungsbeschränkungen für die Bildung der Diskursrepräsentation im Sinne von Klein und Stutterheim (Klein 2007; Stutterheim/Klein 2002) und damit auch für die Sprachproduktion (vgl. Abschnitt 2). Die Beantwortung dieser Frage erfordert offensichtlich ein kognitiv orientiertes, psycholinguistisches Herangehen. Es ist aber nicht auszuschließen, dass tiefgehende qualitative Analysen vergleichbarer authentischer Texte in verschiedenen Sprachen zumindest bei der Hypothesenbildung hilfreich sein können. Wäre das vielleicht eine zukünftige kontrastive Aufgabe für das IDS?

\section{Literatur}

Asher, Nicholas/Lascarides, Alex (2003): Logics of conversation. (= Studies in Natural Language Processing). Cambridge.

Betten, Anne (2011): Kerkerstrukturen. Thomas Bernhards syntaktische Mimesis. In: Knape, Joachim/Kramer, Olaf (Hg.): Rhetorik und Sprachkunst bei Thomas Bernhard. Würzburg, S. 63-80. 
Blühdorn, Hardarik (2007): Zur Struktur und Interpretation von Relativsätzen. In: Deutsche Sprache 35, S. 287-313.

Caroll, Mary et al. (2008): Subordination in narratives and macro-structural planning: a comparative point of view. In: Fabricius-Hansen, Cathrine/Ramm, Wiebke (Hg.): "Subordination“ versus „Coordination“ in sentence and text. A cross-linguistic perspective. (= Studies in Language Companion Series 98). Amsterdam, S. 161-185.

Fabricius-Hansen, Cathrine (2009): Überlegungen zur pränominalen Nicht-Restriktivität. In: Ehrich, Veronika et al. (Hg.): Koordination und Subordination im Deutschen. (= Linguistische Berichte. Sonderhefte 16). Hamburg, S. 89-112.

Fabricius-Hansen, Cathrine (2010a): Adjektiv-/Partizipialattribute im diskursbezogenen Kontrast. In: Deutsche Sprache 38, S. 175-192.

Fabricius-Hansen, Cathrine (2010b): Deutsch im Kontrast - textbezogen. In: Dammel, Antje/ Kürschner, Sebastian/Nübling, Damaris (Hg.): Kontrastive Germanistische Linguistik. Bd. 1. Hildesheim u.a., S. 171-200.

Fabricius-Hansen, Cathrine (i.Dr.): Vorangestellte Attribute und Relativsätze im Deutschen: Wettbewerb und Zusammenspiel. In: Hennig (Hg.).

Gloning, Thomas/Seim, Stefanie (i.Dr.): Attribute und ihre Funktionen in der Literatursprache und in Fachtexten im Deutschen. In: Hennig (Hg.).

Hennig, Mathilde (Hg.) (i.Dr.): Attribution, Komplexität, Komplikation. Berlin.

Holler, Anke (2008): A discourse-relational approach to continuation. In: Benz, Anton/Kühnlein, Peter (Hg.): Constraints in discourse. Proceedings of the First Conference on Constraints in Discourse held at the University of Dortmund. (= Pragmatics \& beyond 172). Amsterdam, S. 249-265.

Holler, Anke (2011): Wie viele nicht-restriktive Relativsätze? Grammatische und pragmatische Indizien. Paper presented at the Workshop „Relativsatz und partizipiale Attribution im Vergleich“, Universität Tübingen.

Kamp, Hans/Reyle, Uwe (2011): Discourse Representation Theory. In: Maienborn, Claudia et al. (Hg.): Semantics. An international handbook of natural language meaning. 1. Halbbd. (= Handbücher zur Sprach- und Kommunikationswissenschaft 33.1). Berlin, S. 872-923.

Klein, Wolfgang (2007) Zwei Leitgedanken zu ,Sprache und Erkenntnis‘. In: Zeitschrift für Literaturwissenschaft und Linguistik 37, 145: Sprachliche Perspektiven, S. 1-34.

Klein, Wolfgang/Stutterheim, Christiane von (1991): Text structure and referential movement. (= Sprache und Pragmatik 22). Lund.

Loock, Rudy (2010): Appositive Relative Clauses in English. Discourse functions and competing structures. (= Studies in Discourse and Grammar 22). Amsterdam.

Lötscher, Andreas (1998): Die textlinguistische Interpretation von Relativsätzen. In: Deutsche Sprache 26, S. 97-120.

Mann, William C./Thompson, Sandra A. (1988): Rhetorical Structure Theory: Toward a functional theory of text organization. In: Text 8, S. 243-281.

Onea, Edgar (2013): Potential questions in discourse and grammar. Göttingen.

Peyer, Ann (1997): Satzverknüpfung. Syntaktische und textpragmatische Aspekte. (= Reihe Germanistische Linguistik 178). Tübingen.

Schlenker, Philippe (2013): Supplements without Bidimensionalism. Expanded version, February 12, 2013. New York. Internet: www.semanticsarchive.net/Archive/jgwMjNmM/ Supplements_without_Bidimensionalism.pdf.

Schmidt, Jürgen Erich (1993): Die deutsche Substantivgruppe und die Attribuierungskomplikation. (= Reihe Germanistische Linguistik 138). Tübingen. 
Solfjeld, Kåre (2004): Zur Wiedergabe deutscher erweiterter Attribute in authentischen norwegischen Übersetzungen. In: Hermes 33, S. 89-116.

Struckmeier, Volker (2007): Attribute im Deutschen. Zu ihren Eigenschaften und ihrer Position im grammatischen System. (= Studia grammatica 65). Berlin.

Stutterheim, Christiane von/Klein, Wolfgang (2002): Quaestio and L-Perspectivation. In: Graumann, Carl F./Kallmeyer, Werner (Hg.): Perspective and perspectivation in discourse. (= Human Cognitive Processing 9). Amsterdam, S. 59-88.

Stutterheim, Christiane von/Carroll, Mary (2007): Durch die Grammatik fokussiert. In: Zeitschrift für Literaturwissenschaft und Linguistik 37, 145, S. 35-60.

Stutterheim, Christiane von/Klein, Wolfgang (2002): Quaestio and I-Perspectivation. In: Graumann, Carl F./Kallmeyer, Werner (Hg.): Perspective and Perspectivation in Discourse. (= Human cognitive processing 9). Amsterdam, S. 59-88.

Stutterheim, Christiane von et al. (2012): How grammaticized concepts shape event conceptualization in language production: Insights from linguistic analysis, eye tracking data, and memory performance. In: Linguistics 50, S. 833-867.

Umbach, Carla (2006): Non-restrictive modification and backgrounding. Paper presented to the Ninth Symposium on Logic and Language, Budapest, 2006.

Weber, Heinrich (1994): Erweiterte Partizipialattribute: Nur eine schriftsprachliche Konstruktion? In: Bresson, Daniel/Dalmas, Martine (Hg.): Partizip und Partizipialgruppen im Deutschen. (= Eurogermanistik 5). Tübingen, S. 149-162.

Zeevat, Henk (2014): Production and interpretation of natural language. Leiden.

Zifonun, Gisela (2001): Grammatik des Deutschen im europäischen Vergleich. Der Relativsatz. (= amades 3/01). Mannheim.

Zifonun, Gisela (2010): Possessive Attribute im Deutschen. In: Deutsche Sprache 38, S. 124-153. Zifonun, Gisela/Hoffmann, Ludger/Strecker, Strecker (1997): Grammatik der deutschen Sprache. (= Schriften des Instituts für Deutsche Sprache 7). Berlin. 